\title{
LA SELECCIÓN DE PERSONAL, ALGUNAS CONSIDERACIONES FRENTE A SUS PRÁCTICAS*
}

\author{
Blanca Yenny Hernández Sánchez ${ }^{* *}$
}

Recibido: octubre 7 de 2010 • Aceptado: mayo 14 de 2012

Nuestra sociedad occidental se caracteriza por una aceptación
acrítica de métodos innobles, siempre y cuando estos métodos
aporten éxito. El valor éxito prima sobre el valor ético y todo
se permite siempre y cuando la violencia ejercida no sea muy
explícita con lo que se permite la instauración de la violencia
psicológica como estrategia para conseguir lo que se desea

(Pares, 2008, p. 368).

\section{RESUMEN}

Existe una arraigada tendencia a complicar los procesos de selección de personal, bajo la premisa de que, de esta manera, serán más científicos, objetivos y eficientes, sin tener en cuenta las implicaciones que los nuevos dispositivos representan para los candidatos que participan en los mismos. El objetivo de este escrito es analizar esta práctica desde una mirada hermenéutica, entendida como posibilidad de leer e interpretar una realidad presente en las empresas, a partir del referente del discurso de la ética y sus implicaciones. Para este efecto, se aplicaron entrevistas en profundidad, a partir de las cuales se pudo constatar que la relación seleccionador-seleccionado (o aspirante al cargo) constituye un ámbito privilegiado de ejercicio de poder que en algunos casos se convierte en dominación.

\section{PALABRAS CLAVE}

Selección de personal, gestión de personal, gestión laboral, ética.

\section{CLASIFICACIÓN JEL}

M12, M54, M55

\section{CONTENIDO}

Introducción; 1. Reflexión en torno a la problemática que entraña este escrito; 2. Algunos aportes a las prácticas de selección de personal; 3. Selección de personal y ética; 4. Algunas evidencias; 5. Conclusiones; Bibliografía.

* Este artículo surge del interés por conocer los efectos que tiene las prácticas de selección de personal en los candidatos que participan en ellas, es de carácter reflexivo y se desarrolla como parte del trabajo de grado de la Maestría en ciencias de la Administración de la Universidad Eafit. Dicho trabajo se elaboró entre los años 2006-2008. La autora pertenece al grupo de investigación Cultura y Gestión Organizacional (CyGO) de la Universidad de Medellín (Categoría B, Colciencias)

** Psicóloga, Universidad San Buenaventura, Medellín, Colombia. Especialista en Gestión Humana, Universidad EAFIT, Medellín, Colombia. Magíster en Ciencias de la Administración, Universidad EAFIT, Medellín, Colombia. Profesora investigadora, Facultad de Ciencias Económicas y Administrativas, Universidad de Medellín, Colombia. Miembro activo del grupo de investigación CyGO y del grupo de investigación de ASCOLFA capitulo Antioquia. Correo electrónico: bhernandez@udem.edu.co, yennyh@une.net.co. 


\section{RECRUITMENT AND SOME CONSIDERATIONS FOR ITS PRACTICE}

\section{ABSTRACT}

There is a common trend by recruiters to make the selection process for personnel more complicated under the premise that the modified processes will be more scientific, objective and efficient. However, this does not take into account the implications these new tools have for the candidates that participate in the selection process. The objective of the paper is to analyze recruitment from the perspective of hermeneutics, understood as the possibility of reading and interpreting a common reality in businesses, starting with the disclosure of ethics and its implications. For this effect, in-depth interviews were conducted from where it could be shown that the relationship between the recruiter and the candidate constitutes a privileged space for the exercise of power which in some cases can turn into an exercise of domination.

\section{KEY WORDS}

Recruitment, personnel management, labor management, ethics

\section{CONTENT}

Introduction; 1. Reflecting on the problem that this paper expresses; 2 . Some contributions for personnel selection; 3. Personnel selection and ethics; 4.Some evidences; 5. Conclusions; Bibliography.

\section{A SELEÇÃO DE PESSOAL, ALGUMAS CONSIDERAÇÕES FRENTE A SUAS PRÁTICAS}

\section{RESUMO}

Existe uma arraigada tendência de complicar os processos de seleção de pessoal, sob a premissa de que, desta maneira, serão mais científicos, objetivos e eficientes, sem ter em conta as implicações que os novos dispositivos representam para os candidatos que participam nos mesmos. O objetivo deste escrito é realizar uma análise desta pratica desde um olhar hermenêutico, entendido como possibilidade de ler e interpretar uma realidade presente nas empresas, a partir do referente do discurso da ética e suas implicações. Para este efeito, se realizaram enquetes em profundidade, a partir das quais se pôs constatar que a relação selecionador-selecionado (ou aspirante ao cargo) constitui um âmbito privilegiado de exercício de poder no qual se incorre, convertendo-se este, em alguns casos, em exercício de dominação.

\section{PALAVRAS CHAVES}

Seleção de pessoal, gestão de pessoal, gestão laboral, ética.

\section{CLASSIFICAÇÃO JEL}

M12, M54, M55

\section{CONTEÚDO}

Introdução; 1. Reflexão em volta à problemática que entranha este escrito; 2. Alguns aportes às praticas de seleção de pessoal; 3. Seleção de pessoal e ética; 4. Algumas evidencias; 5. Conclusões; Bibliografia. 


\section{INTRODUCCIÓN}

La selección de personal es hoy en día un proceso complejo que permite delimitar desde el ingreso el tipo de personal que la empresa desea; además, a partir de la descripción y análisis de cargos, se acomoda a los individuos a los perfiles que la organización construye en razón de las necesidades de la empresa. Visto de una manera superficial y así descrito, no parece tener mayores inconvenientes, puesto que la racionalidad económica presenta como objetivo fundamental, supeditar las necesidades de las personas y de la sociedad misma a las demandas económicas de la firma. Sin embargo, los planteamientos que se encuentran implícitos en las prácticas de selección de personal (los cuales se examinaran en este escrito) presentan bastantes problemáticas cuando se ahonda en las prácticas implicadas en el proceso de escogencia de personal a fin de satisfacer los imperativos que comporta tal racionalidad económica (mayor ganancia, alta eficacia y eficiencia, cero pérdidas: de tiempo y materiales). De tal suerte, que a instancia de las inquietudes manifiestas en el proceso de selección de personal, surge una preocupación por conocer cuáles son las faltas en las que incurre dicha práctica y, además, qué efectos suelen tener estas en las personas que se someten o exponen a ellas.

Desde una mirada hermenéutica, se estableció un acercamiento al objeto de estudio aquí expresado (las prácticas de selección de personal); para este efecto se hicieron entrevistas de profundidad' a personas que han participado en procesos de selección en empresas de la ciudad de Medellín; las entrevistas incluían personas que fueron elegidas y otras que no pasaron dicho proceso². El análisis de la información se realizó desde la hermenéutica a partir del análisis del discurso; la interpretación y el análisis de los contenidos fueron triangulados desde teóricos como Hortal (2002), Pena (2004), Cortina (1985 y 2005) y Foucault (1980 y 2005), entre otros.

Por otro lado, existe una posibilidad de abordar las prácticas de selección de personal, vinculada con una tendencia cada vez más necesaria y pertinente en la

1 En el marco del trabajo de campo se realizaron 2 grupos focales y 6 entrevistas en profundidad, se utilizó el método etnográfico, en particular, el concepto de entrevista planteado por Guber (2001), quien reconoce el carácter reflexivo de la entrevista, en el cual entrevistado y entrevistador logran establecer un espacio de confianza; en este sentido, entonces, se reconoce el diálogo como la posibilidad de construcción de sentido, coherente con el carácter cualitativo-hermenéutico de la investigación. Dichas entrevistas fueron aplicadas a personas que pasaron por procesos de selección; algunas fueron contratadas y otros rechazados en las empresas que realizaron el proceso; la proporción aproximada entre los contratados y los rechazados fue del $50 \%$. La selección de los entrevistados estuvo vinculada a referidos, de igual forma a personas conocidas por la investigadora (aprovechando el rol que ella misma, para aquel entonces ejercía como psicóloga de selección en algunas empresas de Medellín), en ambos casos debían cumplir con la condición de haber pasado por procesos de selección de personal complejos, todos los entrevistados fueron de la ciudad de Medellín, en su mayoría participaron en procesos de selección en empresas privadas, al menos dos de ellos en empresas públicas y uno en un proceso de una organización caza talentos.

2 En el anexo A se presenta la guía utilizada en las entrevistas para el trabajo de campo. 
vida organizacional; se hace referencia aquí a la ética como discurso vinculante y posibilidad de virar la condición de quienes realizan los procesos de selección de personal, además, de quienes participan en calidad de candidatos a seleccionar.

Es así como se abordan discursos como el de Hortal (2002) y Cortina (1985, 2005), en los cuales se encuentra sintonía con respecto a los elementos que aquí se tratan. Desde el primer autor es posible entender la ética de las profesiones, para el caso que aquí compete, el de los profesionales en psicología, encargados de la mayoría de los procesos que se llevan a cabo en la selección de personal, y desde la segunda autora es posible construir sentido, toda vez que aborda la preocupación por la ética en la organización, en tanto un compromiso con las acciones, reflejadas en el contexto social. En este sentido la autora aboga por la acción virtuosa de las organizaciones, establece una diferencia entre virtudes y vicios, de tal suerte que las organizaciones éticas deberían optar por las virtudes en tanto les permiten actuar desde la ética; al respecto Cortina $(2005$, p. 2) menciona: "Pero en general ¿qué son las virtudes? Son las predisposiciones para actuar con justicia, prudencia, honestidad, etc., y ¿qué son los vicios? Pues lo contrario, la predisposición para actuar con injusticia, imprudencia, opacidad, deshonestidad, etc.".

Se propone, entonces, cuestionar desde una perspectiva hermenéutica, un proceso que hace parte de la gestión humana y del quehacer administrativo, además de tener los fundamentos arraigados en la psicología, en tanto toma algunas herramientas propias de esta disciplina y en ocasiones hace uso de ellas de manera descontextualizada. Se tratará en adelante sobre las prácticas en las que incurre la selección de personal en tanto proceso cuyo propósito es el de cumplir los requerimientos de organizaciones productivas.

En el inicio de este escrito se presentan algunas reflexiones sobre la problemática del proceso de selección de personal; acto seguido se exponen algunos aportes de autores que se han ocupado de indagar acerca de las prácticas de selección de personal, para cerrar con conclusiones que se abordan desde la reflexión, acompañadas de autores como Foucault (1980 y 2005), Deleuze (1990) y Cortina (1985 y 2005), autores que permiten en algunos casos contextualizar el problema, y en otros, sustentan la postura ética que se encuentra inmersa en este escrito.

\section{REFLEXIÓN EN TORNO A LA PROBLEMÁTICA QUE ENTRAÑA ESTE ESCRITO}

La experiencia desde el espacio profesional ha permitido a partir del conocimiento y práctica de la selección de personal, asumir una postura crítica frente a esta. En tal acción se han podido percibir problemas que se relacionan con el ámbito ético del quehacer de la organización, en particular con aquellas personas que se ven implicadas en el proceso, so pretexto de identificar en ellas el perfil ideal 
(promesa que no pasa de ser promesa) y que, a la postre, deviene sometimiento y/o abusos psicológicos.

Similar a como ocurre con la administración, en donde se encuentra una marcada necesidad por acogerse a las prácticas de gestión del momento (la mayoría de ellas traídas desde contextos foráneos, de difícil aplicación en nuestra cultura), López $(1998,1999)$ nombra tales estrategias organizacionales como "modas administrativas" las cuales suelen ser utilizadas para efectos de manipulación y control en las empresas. Estableciendo una comparación se podría decir entonces que la selección de personal es una práctica de la psicología aplicada muy cercana a la adhesión a las modas. En dicha práctica es frecuente la utilización de artefactos que ayudan a establecer parámetros de validez en las respuestas de los interrogados — que no evaluados-, como el caso del polígrafo, la grafología y los centros de simulación, entre otros.

Por otro lado, se hace referencia a un fenómeno que atañe en forma particular al ejercicio de los administradores, vinculado en ocasiones de forma directa con la práctica de selección de personal, dado que ellos mismos se convierten en ejecutores de parte del proceso de selección, cuando no del proceso en su totalidad.

La postura crítica se halla acompañada de una preocupación de carácter ético que permite, desde el discurso comprensivo, reflexionar en torno a un hecho que se encuentra enmarcado en una de las ciencias o disciplinas en las que la administración se ha apoyado, a saber, la psicología.

Por otra parte, es pertinente para el ejercicio docente, la construcción de estos espacios de reflexión que permitan establecer denuncias en el aula de clase, no desde la sospecha y el malestar, sino desde el conocimiento, mirado en tanto constituye una conversación entre organización, psicología y administración, visto, en fin, desde el conocimiento interdisciplinario, ya que atañe tanto a los psicólogos que participan de la psicología aplicada a la empresa, como a los administradores, y de forma particular a los docentes que participan en la formación de unos y otros.

En este orden de ideas, el propósito es interpelar las prácticas de selección de personal. También avanzar algunas conjeturas en torno a un ejercicio profesional poco abordado en el sentido crítico. Es así como a partir de este análisis surgen interrogantes alrededor de estas acciones, tales como los que siguen: ¿Qué se oculta tras las prácticas, procedimientos y dispositivos que de forma continua emplea la selección de personal en las organizaciones? ¿Qué tan neutrales y objetivas pueden ser consideradas las prácticas y procedimientos empleados en los procesos de selección de personal? ¿Qué efectos suelen producir en el sujeto (candidato) las prácticas, procedimientos y dispositivos que emplea la selección de personal en 
las organizaciones? ¿Qué tan inocuos o respetuosos de la dignidad de la persona resultan ser los dispositivos y prácticas de los que se sirve el proceso de selección de personal sobre el sujeto (candidato) a un puesto o cargo?

\section{ALGUNOS APORTES A LAS PRÁCTICAS DE SELECCIÓN DE PERSONAL}

Cada vez son más las voces que interpretan e interrogan las prácticas que hacen parte de la selección de personal, aunque en el contexto local no abundan las publicaciones en este sentido. Se encuentran estudios que han estado orientados a conocer la percepción de los candidatos ante el proceso de selección como el de Osca y García (2004); también un trabajo relacionado con la desprotección de la vida privada del candidato, de Lievens (1984), además de Montmollin (1999), autor francés que presenta los "Psicofarsantes", texto en el cual el autor se va lanza en ristre contra los profesionales en psicología y las prácticas que ejecuta en la organización, incluida la selección de personal ${ }^{3}$.

Respecto a la perspectiva ética de las organizaciones, elemento que resulta relevante para el análisis que ocupa este escrito, se encuentra un trabajo interesante presentado por Ting-Ding y Déniz (2007). En dicho trabajo, Ting-Ding y Déniz (2007, p. 58), se preocupan por analizar las implicaciones éticas de la selección de personal, toda vez que los procedimientos de selección pueden llegar a afectar el bienestar psicológico de los participantes; el decir de los autores es que dichos efectos pueden llegar a tener una larga duración; como ejemplo mencionan a los participantes que perciben la injusticia en los test de selección, situación esta que puede llegar a afectar la autoestima de los candidatos especialmente en aquellos que han sido rechazados en el proceso.

Por otro lado, Ting-Ding y Déniz (2007), en el mismo trabajo, plantean que en la entrevista de selección se establece una relación de poder, de tal suerte que dicho ejercicio de poder puede terminar generando abuso, en tanto la posibilidad de que en ellas se someta a estrés innecesario, en este sentido Ting-Ding y Déniz (2007, p. 65) afirman:

[...] en el caso de la entrevista nos encontramos con una relación de poder en la que generalmente el entrevistador posee algo que el entrevistado quiere; aunque la medida en que el entrevistado domine probablemente variará de acuerdo con la cantidad y calidad de los candidatos. De manera inevitable, donde hay poder existe la posibilidad de abusar de él, emprendiendo comportamientos no éticos y violando así los derechos del candidato. Además es probable que carezca de utilidad someter a los candidatos a estrés de manera innecesaria, ya que la evidencia sugiere que la manera en que son tratados los candidatos afecta su disposición a aceptar las ofertas de trabajo.

Sobre estos antecedentes se amplía en el artículo de Hernández (2009). 
Respecto a la entrevista de selección, además, plantean cómo algunas preguntas no están orientadas hacia temas relevantes para el trabajo, en ocasiones se desvían hacia información sobre la vida privada, ideologías, ocio, aficiones, Dtendencia sexual, hábitos de consumo, entre otras. dicha información puede llegar a sesgar o a generar prejuicios que van en contra de la idoneidad del candidato para ocupar un puesto de trabajo, aspectos estos que en ningún caso son relevantes para la empresa y, sin embargo, sí van en contra de las personas y su derecho a la protección de la intimidad, Ting-Ding y Déniz (2007)

A partir de los planteamientos establecidos por los autores aquí citados y el interés que se ha puesto en evidencia en este escrito, se aborda en adelante la ética como elemento de discusión frente a las prácticas de selección de personal.

\section{SELECCIÓN DE PERSONAL Y ÉTICA}

Como ya se ha establecido, en este análisis se muestra un paralelo entre la ética y las prácticas de selección de personal; en este sentido se encuentra un autor que ha ahondado en el discurso de la ética en los negocios; se hace alusión aquí a Pena (2004), quien argumenta sobre los tres principios fundamentales de la ética: 1) no hacerme daño yo mismo, 2) no permitir que otros me hagan daño y 3) no hacerle daño a otros. Se puede entonces afirmar que el abuso sobre el otro presente en ocasiones en la selección de personal, es una suerte de ejercicio que va en contra de la ética, toda vez que viola el tercer principio fundamental.

Por otro lado, las pruebas de las que se vale el proceso de selección son, en gran medida, validadoras de algunas características de personalidad, de conocimientos, de capacidades o competencias, como se suelen nombrar en la actualidad; sin embargo, lo que se ha encontrado, a partir de algunos procesos de selección y de la percepción de los mismos candidatos participantes en dichos procesos, no es en efecto esa búsqueda de las capacidades, aptitudes, virtudes, lo que indaga la selección de personal. En la mayoría de los casos es todo lo contrario, se busca la falta, la falla, la mentira, la debilidad del candidato; la relación se establece entonces desde la desconfianza, toda vez que pretende fiscalizar dónde está el error, dónde el otro se equivoca, cuál es la patología que esconde; prueba de ello son las entrevistas, en las cuales de forma repetitiva se realizan preguntas que se repiten, verbalizadas de forma diferente, para detectar dónde el candidato se contradice.

De igual manera, cuando se llevan a cabo las visitas domiciliarias, se analizan, más que las relaciones sociales, las condiciones de lujo, con quién vive, qué consume, cómo ordena la casa, si es soltero o casado, si se puede verificar la información que estableció en todo el proceso de selección; ante todo, que no vaya a ser delincuente y de una vez se definen las inclinaciones o 'aberraciones' sexuales. Lo expuesto ratifica la ausencia de ética en las prácticas de selección de personal. 


\section{ALGUNAS EVIDENCIAS}

Es posible, entonces, puntualizar sobre algunas conjeturas que se desprenden del análisis de las prácticas de selección de personal. En este sentido y respecto a la pertinencia de la mirada ética en dichos procesos, anotar la presencia de ejercicio de dominación en términos de poder. Se evidencia en este proceso, según los planteamientos de Foucault (1980), un ejercicio de dominación en tanto el poder es ejercido sobre las personas; de igual forma, la utilización de artefactos o dispositivos de poder, designados por Deleuze (1990).

Si se parte de lo que Deleuze (1990) define como dispositivo, es pertinente virar sobre el caso de los centros de simulación (assessment center); esta herramienta, bastante utilizada en los procesos de selección de personal en la actualidad, es definida por Díaz (2001) como espacio de observación o de vigilancia. Acude pues a convertirse en un nuevo y sofisticado panóptico5; de la misma forma, los test psicotécnicos regulan, controlan, estandarizan y separan; son dispositivos que perpetúan este ejercicio de dominación, de un saber, un conocimiento sobre el otro;

4 Expresa Díaz (2001) que los orígenes de los centros de simulación corresponden a fines militares, específicamente en la Alemania de la pre-guerra, en donde seleccionaban oficiales destinados a misiones riesgosas; estos procedimientos se trasladaron también a la Junta Británica de Guerra de Selección Oficial (WOSB ), con los cuales se sustituyen los sistemas de entrevistas cortas por las reuniones de evaluación. La WOSB continuó operando y constituye el lazo histórico directo de otros centros de evaluación del sector público británico.

Según Díaz (2001, p. 263), los centros de simulación tienen un remanente en EE. UU. a donde llegan: "(...) en los días de la II Guerra Mundial, cuando la Oficina de Servicios Estratégicos (OSS) del gobierno tenía necesidad de seleccionar individuos capacitados para realizar misiones arriesgadas de inteligencia secreta, poco usuales y contrató a muchos que resultaron ser inadaptados". Es así como vinculan la colaboración de la psicología al proceso de selección de soldados, se identifica como creador del procedimiento al psicólogo Douglas Bray, quien elaboró la primera evaluación hacia finales del año 1943"

5 En la obra de Foucault (1980), se encuentra la referencia al panóptico, como dispositivo por excelencia de las sociedades disciplinarias; se trata entonces de una disposición arquitectónica creada por Jeremy Bentram en 1791, desde la cual se puede observar a $360^{\circ}$ lo que ocurre en un centro de encierro determinado. Respecto del panóptico, Foucault (1980, p. 17) menciona:

El principio era: en la periferia un edificio circular; en el centro una torre; ésta aparece atravesada por amplias ventanas que se abren sobre la cara interior del círculo. El edificio periférico está dividido en celdas, cada una de las cuales ocupa todo el espesor del edificio. Estas celdas tienen dos ventanas: una abierta hacia el interior que se corresponde con las ventanas de la torre; y otra hacia el exterior que deja pasar la luz de un lado al otro de la celda. Basta pues situar un vigilante en la torre central y encerrar en cada celda un loco, un enfermo, un condenado, un obrero o un alumno. Mediante el efecto de contra-luz se pueden captar desde la torre las siluetas prisioneras en las celdas de la periferia proyectadas y recortadas en la luz. En suma, se invierte el principio de la mazmorra. La plena luz y la mirada de un vigilante captan mejor que la sombra que en último término cumplía una función protectora.

Expresa Foucault que antes de Bentham esta preocupación existía ya. Menciona como un ejemplo de ello la escuela militar de París en 1755 respecto a la disposición de los dormitorios, en donde los alumnos debían disponer de una celda con cristales a través de la cual podían ser vigilados durante la noche de tal manera que no pudiera tener contacto con otros compañeros o con la servidumbre. 
La selección de personal, algunas consideraciones frente a sus prácticas

la entrevista cumple de igual manera con una función específica de los dispositivos: "máquina para hacer ver y para hacer hablar".

Se puede comprobar en relatos que fueron obtenidos mediante entrevista ${ }^{6}$ a personas que han pasado por proceso de selección, evidencia de cómo algunos elementos que hacen parte del proceso de selección, pueden ser utilizados como dispositivos, al respecto el Entrevistado 1 (2007) opina:

[...] ella siempre mira fijo, como tratándolo de intimidar a uno; -más adelante comenta- y ellas son preguntándote cosas y te ponen cascaritas y te dicen sí, pero, entonces qué es lo más importante. Usted respondió que lo más importante es el volumen de la empresa, entonces volumen mata rentabilidad. ..o sea, ellas te empiezan o poner cascaritas y entonces es ahí donde la gente se asusta, ahí es donde la gente empieza como a tomárselo personal; dice también: y ellas dos son sentadas al frente mirando uno como se relaciona, quién es el que lleva el liderazgo, quién es la más terca, todo, ahí están mirando todo.

Respecto a un artefacto que, como tal, es también, según Deleuze (1990), un dispositivo, a saber, el polígrafo, se encuentran testimonios como el que relata el Entrevistado 3 (2007):

En el polígrafo sí sentía como que estaban mirándome muy allá, o sea, yo decía: pero por qué me hacen esa clase de preguntas, me preguntan que si yo había robado, que si yo tenía un contacto o nexo con el narcotráfico; son preguntas que comúnmente a uno no le hace nadie, o sea, yo no creo que a vos te hayan preguntado, iAh!, vos sentís..., y uno dice: ve y este por qué me está preguntando eso, y bueno y si yo soy gay o me gustan otras personas, ¿̇qué? (...).

El polígrafo, en tanto dispositivo concebido y diseñado para el sometimiento del cuerpo, da cuenta de una práctica o ejercicio de poder, de una relación asimétrica de miradas; al respecto, Foucault (2005) menciona que el control del cuerpo ejercido desde el poder se constituye en una política de coerción, de manipulación, en la que se calculan los gestos, los comportamientos, en este sentido el cuerpo humano se explora, se desarticula y recompone. En palabras de Foucault (2005, p. 141), se ejerce una suerte de 'anatomía política' que es análoga a una 'mecánica del poder' a través de la cual se hace presa el cuerpo de los otros, "(...) no simplemente para que ellos hagan lo que se desea, sino para que opere como se quiere, con las técnicas, según la rapidez $y$ al eficacia que se determina".

Por otro lado, en referencia a los abusos, que tienen presencia en los procesos de selección de personal, se hacen evidentes algunos relatos de entrevistados, situaciones que pueden verse como sensaciones de intimidación o ridiculización,

6 En el marco de este trabajo se realizaron entrevistas abiertas a profundidad a personas que han pasado por procesos de selección de personal, con el propósito de garantizar obtención de información relevante y de interés. Se garantizó a los entrevistados confidencialidad sobre la información personal; por esta razón no aparecen los datos de los mismos. 
Respecto a estos abusos se puede citar una verbalización que hace el Entrevistado 1 (2007) que ha participado de un proceso de selección de personal, el cual ha durado un año y medio.

[...] un abuso es esto por ejemplo mío, que es un abuso que lleva un año y medio, eso es un abuso porque es que si a usted le están diciendo... bueno usted lleva un año y medio, que lo llamen, que le expliquen, o sea, ellos tienen que hacer más seguimiento y más control de toda una cantidad de cositas chiquiticas que tienen alrededor, que para ellos es muy normal porque día a día hacen doscientos mil, pero para uno no, porque uno es el implicado uno es el doliente.

De igual manera, como se mencionó en el inicio de este escrito, en los postulados de Cortina (2005) se encuentran posibles orientaciones para propender por una selección de personal más ética, vinculada al deber ser de las organizaciones. Si estas logran, entre otras cosas, realizar procesos más respetuosos con la dignidad de las personas, que se orienten a lo que Cortina (2005) menciona como la necesidad de contribuir a la construcción de empresas más éticas, toda vez que la ética de la empresa da cuenta del carácter de las empresas, de cómo y en qué sentido deben estas forjar un carácter, si las empresas logran esto, afirma Cortina (2005, p. 4) siguiendo a Ortega y Gasset, estarían 'altas de moral', entendiendo la moral como estar alto de moral o desmoralizado, no desde el par moral-inmoral; para una definición más clara afirma:

Importa estar altos de moral, nadie quiere estar bajo de moral o desmoralizado, porque cuando te encuentras bajo de moral o desmoralizado, no tiene ganas ni siquiera de vivir, de ser proactivo o de tomar decisiones. El alto de moral se anticipa al futuro, es proactivo, lo crea, intenta ganarle la mano, porque tiene la moral alta.

Además del análisis que aquí se ha planteado, respecto a la connotación de abuso, se evidencia en Rodríguez y Cols (2005) sintonía con lo que aquí se ha tratado de expresar, toda vez que reconoce que el abuso es facilitado por la capacidad de poder que la parte que abusa tenga a priori sobre la otra parte.

De igual manera, en este escrito se logra un análisis desde un acercamiento hermenéutico, en tanto como lo plantea Vásquez (2004), la hermenéutica centra su preocupación en la comprensión del conjunto, en ella habita pues una preocupación por explicar fenómenos que en ocasiones escapan a la mirada desprevenida, que permite interrogarse más allá de lo evidente y que cobra sentido a partir de la lectura del investigador.

\section{CONCLUSIONES}

Según los relatos de algunos entrevistados, se evidenciaron abusos como intimidación mediante la utilización de artefactos como el polígrafo, los centros de simulación (assessment center), demoras excesivas en el proceso de selección, as- 
pectos que dan cuenta de situaciones desafortunadas que las más de las veces los participantes en estos procesos no denuncian por distintas razones: una de las más importantes es la falta de legislación clara sobre estas prácticas, la ley colombiana está en mora de reglamentar sobre estos procesos; otra podría estar referida al temor (algunas veces imaginario) a las represalias que las organizaciones pueden llegar a tener si sus empleados critican la forma como fueron seleccionados; otra razón que resulta ser muy evidente es el nivel educativo y la desinformación de los candidatos respecto a lo que se puede o no hacer en estos procesos.

Es posible pensar que la selección de personal es un proceso que se hace necesario en las organizaciones, toda vez que la empresa requiere identificar competencias laborales propias del personal que ingresa a la empresa; sin embargo, complicar el proceso con artefactos como el polígrafo, los centros de simulación, entrevistas intimidantes, no resulta ser la solución, máxime cuando este en ocasiones puede llegar a violar principios éticos fundamentales. La reflexión entonces no está orientada a proponer que dichos procesos desaparezcan; más bien se trata de contribuir a lograr que estos sean más respetuosos y acordes con lo que la empresa requiere conocer de quien aspira ingresar a la misma, sin violar la privacidad y autonomía de los candidatos.

Finalmente, el propósito de este escrito no ha sido otro que buscar un acercamiento a los procesos de selección de personal desde una mirada crítica y reflexiva, en un afán por propender por formas más dignas de establecer un primer contacto con las organizaciones mediante los procesos de selección de personal; acudir entonces en este caso, a algunos autores que se han ocupado de la ética en las organizaciones tiene un propósito explícito, y no es otro que plantear la necesidad de promover relaciones de respeto en la dupla seleccionador-seleccionado, en donde no se vulneren los derechos de las personas debido a la condición de poder que ejerce aquel que ofrece el empleo, situación que lo habilita para realizar procesos de selección en ocasiones ambiguos e irrespetuosos, sin tener en cuenta las consecuencias que ello pueda tener en los participantes. Es esta una posibilidad de poner en contexto prácticas que en el medio colombiano en general y de Medellín en particular han sido poco abordadas y las más de las veces aceptadas de forma acrítica.

\section{BIBLIOGRAFÍA}

Cortina, A. (1985). Razón comunicativa y responsabilidad solidaria. Salamanca: Ediciones sígueme, 187p.

Cortina, A. (2005). La ética de la empresa. Conferencia con USEM. México, junio.

Deleuze, G. (1990). ¿Qué es un dispositivo?» en Michel Foucault, filósofo. Barcelona: Gedisa, 155p. 
Díaz, M. (2001). La GRH como potencialidad individual y su evaluación a través del assessment center : En: Revista Cubana de Psicología, Universidad de la Habana, Vol. 18, No. 3, p. 259-270.

Foucault, M. (1980). El ojo del poder. Entrevista con Michel Foucault En: Jeremías Bentham, El Panóptico. Madrid: La Piqueta, p. 9-26

Foucault, M. (2005). Vigilar y Castigar. 34 ed., México: Siglo veintiuno, 300p.

Guber, R. (2001). La etnografía. Método, campo y reflexividad. Medellín: Norma. 146p.

Hernández, Y. (2009). Prácticas de selección de personal, un instrumento de dominación socialmente aceptado. En: Revista Ad- Minister, Universidad Eafit, No. 14, enero-junio, p. 137-160.

Hortal, A. (2002). Ética general de las profesiones. Bilbao: Editorial Desclée, 278p.

Lievens, S. (1984). La protección de la vida privada en la selección de personal. En: Revista Latinoamericana de Psicología. Vol. 16 No. 1, p. 17-24.

López, F. (1998). Educación en administración y modas administrativas en Colombia. En: Revista Universidad Eafit. Julio-agosto-septiembre. NO. 109, p. 59-88.

López, F. (1999). Prescripciones administrativas en boga ¿cantos de sirena? En: Revista Universidad Eafit. No. 115, p. 9-26.

Montmollin, M. (1999). Los Psicofarsantes. España: Editotial Siglo XXI, 112p.

Osca, A. y Garcia, L. (2004). ¿Cómo perciben los candidatos los procesos de selección ? Una aproximación desde el modelo de Justicia Procedimental de Gilliland. En: Revista de Psicología del Trabajo y de las Organizaciones. Vol. 20, No. 2, p. 225-247.

Pares, S. (2008). La administración de las organizaciones y el acoso moral. En Rafael Carvajal (Editor) En: Gestión Crítica Alternativa. Cali: Universidad del Valle, p. 367-409.

Pena, R. (2004). La empresa ética: ¿un nuevo paradigma? Condiciones, desafíos y riesgos del desarrollo de la business ethics Tesis doctoral. Universidad Complutense de Madrid.

Rodríguez, Á. y Cols. (2005). Un estudio comparativo de las estrategias de abuso psicológico: en pareja, en el lugar de trabajo y en grupos manipulativos. Facultad de Psicología, Universidad de Barcelona, En: Revista: Anuario de Psicología, Vol. 36, No. 3, p. 299-314.

Ting-Ding, J. y Déniz, M. (2007). La selección de personal como un proceso ético y eficiente. El caso de la entrevista de personal. Conocimiento Innovación y emprendedores: camino al futuro, p. 3555-3571.

Vázquez, F. (2004). La cultura como texto: lectura, semiótica y educación. $2^{\mathbf{a}}$ ed., Bogotá: Editorial Javegral, 252p.

\section{Entrevistas}

Entrevistado 1 (2007). Entrevista realizada a una persona que participó en un proceso de selección para una empresa caza talentos, Medellín, 17 de agosto.

Entrevistado 3 (2007). Entrevista realizada a una persona que participó en un proceso de selección para una empresa aeronáutica, Medellín, 17 de octubre. 


\section{ANEXO A. \\ FORMATO GENERAL DE LAS ENTREVISTAS PARA EL TRABAJO DE CAMPO DE LA INVESTIGACIÓN: “LAS PRÁCTICAS DE SELECCIÓN DE PERSONAL, IMPLICACIONES EN LOS ASPIRANTES"}

OBJETIVO: Construir una conversación, en donde los entrevistados y la entrevistadora, logren un diálogo en torno a los procesos de selección en los que han participado, con el fin de identificar posibles abusos que den cuenta del objeto de estudio de la investigación.

\section{- Primer momento: información del actor social}

1. Presentación y saludo

2. Encuadre: contextualización del trabajo de grado que se está llevando a cabo para la Msc. Y la pertinencia de la información que los entrevistados puedan suministrar para tal fin. Espacio abierto para posibles preguntas que los entrevistados consideren pertinentes, con el fin de establecer un ambiente de confianza y tranquilidad.

Tiempo de la entrevista: 45 min a una hora aproximadamente,

3. Datos biográficos:

Nombre:

Edad:

Estado civil:

Profesión:

4. Preguntas orientadoras:

He sabido que usted ha participado de algunos procesos de selección, me podría decir ¿cuántos han sido?, ¿podría hablarme de cada uno de ellos? Empezar por el primero y orientar la entrevista de tal forma que hable independientemente de cada uno.

Tener en cuenta durante la entrevista preguntar sobre la caracterización de los abusos:

Demoras en el proceso y jornadas extenuantes: ¿Cuánto tiempo tuvo para cada examen o prueba?, y en síntesis, ¿cuánto tiempo duró todo el proceso? 
Intimidación: ¿qué sensaciones o sentimientos despertaron en usted la observación y el control de quienes dirigieron el proceso selección?

Ridiculización: hubo momentos en los cuales tuvo que realizar, dramatizaciones, juegos, exposiciones ¿los describe por favor? ¿Cómo se sintió en ese momento? ¿Qué opinión le merecieron en su momento tales prácticas?

Evocación de situaciones traumáticas: respecto a la entrevista particular o grupal, chubo momentos en los que se le interrogó sobre su vida privada? ¿En las conversaciones que tuvo con quienes la entrevistaron, establecieron resultados de las pruebas con aspectos de su vida familiar o de su vida privada? ¿Recuerda algo que la hubiese incomodado?

Sobre las formas de selección de personal: Preguntar directamente al final de la entrevista, qué opina la entrevistada de los procesos de selección de personal y las nuevas tecnologías que se han ido vinculando al mismo (polígrafo, centros de simulación, entre otros).

\section{- Segundo momento: percepción y sentimientos (pasados y presentes)}

Que sentimientos y percepción tuvo y tiene respecto a los procesos de selección en los que ha participado.

¿Podría decirme lo que sintió durante los procesos de selección en los que participó?

¿Qué percepción tuvo de ellos?

¿Qué percepción tiene ahora de ellos? ¿Qué emociones le despiertan?

Hipótesis: la otra cosa que me interesa además de lo que usted me ha contado hasta el momento son los abusos en la selección de personal ¿Eso le dice algo? ¿Quiere decir algo al respecto? o ¿Quiere agregar algo sobre esto, que haya caído en la cuenta en ese momento?

\section{- Tercer momento: cierre}

Dar por terminada la entrevista, agradeciendo la oportunidad y comprometiéndome a hacerle llegar la información una vez analizada en el trabajo de grado, con el fin de que conozca cómo fue analizada la información que me brindaron. 